\title{
Les gardiens des politiques de santé face à la réforme Obama
}

\author{
William GENIEYS, Mohammad-Saïd DARVICHE, Brent EPPERSON
}

\section{Résumé :}

L'étude de la réforme de l'assurance maladie aux États-Unis durant les années 1990 et 2000 montre que loin de disparaître, l'influence des élites de l'État fédéral a été reconfigurée. À l'image des «custodians of policy » de Philip Selznick, de petits groupes d'élites professionnalisés durablement au cœur des pouvoirs exécutif et législatif ont acquis une expertise stratégique dans certains secteurs de politique. Comme leurs homologues français, les élites des politiques de santé étasusiennes se sont posées en gardiens des politiques de santé en adaptant l'impératif du contrôle des coûts à un héritage historique, institutionnel et politique défavorable à une réforme alignant le système d'assurance maladie sur le modèle des autres démocraties occidentales. C'est au prix de ce «provincialisme» et de la recherche invétérée d'un arrangement politique que la réforme Obama a pu aboutir à une évolution du sytème d'assurance maladie qui, à défaut d'être systémique, n'en est pas moins la plus importante depuis les années 1960.

\section{Mots-clés :}

CONTROLE DES COUTS, EliTES, GARDiEN DES POLITIQUes, PoliCy STATE, REFORME POLITIQUe, SECTEUR SANTE, TRAJECTOIRES PROFESSIONNELLES

\begin{abstract}
:
The study of American health care reform during the 1990s and 2000s reveals that, far from waning, the influence of federal policy elites has been reconfigured. Like Philip Selznick's “custodians of policy," small groups of elites with long-term careers at the heights of power in the executive and legislative branches have acquired strategic expertise in key policy sectors. Like their French counterparts, these health policy elites positioned themselves as guardians of health policy by adapting the imperative of cost control to a historical, institutional, and political legacy-one that stands in opposition to comprehensive systemic reforms that would align the U.S. with the prevailing models of universal access in other western democracies. It is at the cost of this "provincialism" and the determined quest for a political compromise that Obamacare succeeded in implementing changes which, despite not amounting to comprehensive systemic reform, nonetheless stand out as the most important American health care reforms since the 1960s.
\end{abstract}

Keywords :

CAREER TRAJECTORIES - COST CONTROL - CUSTODIAN OF POLICY - ELITES - HEALTH SECTOR - POLICY STATE - POLITICAL REFORM 
Même si, dès les années 1980, le tournant néo-libéral a contesté l'emprise technocratique des élites d'État ${ }^{1}$ sur les politiques dans l'ensemble des démocraties (Suleiman, 2003) et, en particulier, aux États-Unis, le policy state (Orren, Skowronek, 2017) ${ }^{2}$ continue son déploiement par-delà les clivages partisans (Jacobs et al., 2019). Un certain nombre d'études récentes suggèrent que le rôle de ces élites en tant qu'acteurs des réformes d'assurance maladie resterait important, même si leur capacité d'action a dû être progressivement reformulée dans certains domaines de politiques publiques (Genieys, 2010 ; Hassenteufel et al., 2010 ; Genieys, Hassenteufel, 2012). De notre point de vue, en raison de leurs propriétés socioprofessionnelles et de l'environnement (culturel, institutionnel, etc.) dont elles sont issues et auquel elles contribuent, ces élites sont en capacité de reformuler les idées et pratiques néo-libérales.

Notre attention porte plus particulièrement sur les élites qui occupent, à l'intérieur de l'État, l'espace où se rencontrent expertise, alignements partisans, intérêts particuliers et contraintes institutionnelles. De fait, leur rôle va bien au-delà de celui de simples experts conseillant les hommes politiques dans la mesure où elles sont «en charge » de la conception même des politiques étatiques. En dépit de l'éclatement des institutions occupées par les experts aux États-Unis (Lepont, 2016 a et b), des élites spécialisées dans le gouvernement des politiques (policy elites) ont, comme en France ${ }^{3}$, développé des caractéristiques combinant expertise technique et connaissance des enjeux politiques, institutionnels et sociaux. Leur singularité professionnelle est le résultat d'une longue expérience des coulisses du pouvoir, profondément ancrée dans un secteur de politique et une forte identification à des programmes de politique réaffirmant la capacité régulatrice de la puissance publique (Genieys, Joana, 2015). Dans cette perspective, on peut supposer que le problème chronique de gestion du système d'assurance maladie a pu, tout comme en France (Genieys, 2010; Genieys, Hassenteufel, 2015), favoriser au niveau fédéral de l'État américain l'émergence d'un nouveau type d'élites.

Dès lors, il semble pertinent d'appréhender la recomposition des sommets de l'État américain, depuis les années 1990, en établissant une corrélation entre transformation de la structure professionnelle des élites et orientation des politiques publiques fédérales en matière d'assurance maladie. Les deux projets américains de réforme de l'assurance maladie, le Clinton Plan (1993-94) et celui qui aboutira à l'ACA (Affordable Care Act) (2008-10), permettent relier les changements survenus dans le profil sociologique des élites washingtoniennes de la santé à la transformation de l'offre programmatique réformatrice (Genieys, 2020). Après une rapide évocation des caractéristiques socioprofessionnelles de ces élites pour la période étudiée (1988-2010) ${ }^{4}$, nous analyserons les liens pouvant être établis entre leur formation spécialisée dans les politiques

\footnotetext{
${ }^{1}$ Par « élites d'État », nous entendons celles qui, après la Deuxième Guerre mondiale, ont adopté le rôle d'architectes de la planification, à l'interface du gouvernement, du parlement et de l'administration, pour développer des styles de politiques variant d'un État à l'autre (Genieys, 2010).

2 Pour Karen Orren et Stephen Skowronek, le policy state est la forme historique prise aux États-Unis, par l'interventionnisme étatique. Ce dernier se caractérise par la prolifération des politiques publiques dans le cadre d'un pouvoir central traditionnellement doté d'une faible légitimité et capacité d'action (Darviche, Genieys, 2018 ; Benoît, Bezes, 2019).

${ }^{3}$ En opposition au « quasi-monopole de l'expertise légitime » des hauts fonctionnaires en France (Béland, 2019).

${ }^{4}$ Cet article s'appuie sur le matériel empirique collecté avec le programme OPERA (Operationalizing Programmatic Elites Research in America) financé par l'Agence nationale de la recherche de 2008 à 2012. Le projet concernait la transformation des structures gouvernantes des États-Unis notamment dans le secteur de la santé de 1988 à 2010. Le projet a permis la production d'une base de données de 152 fiches biographiques détaillées et 191 entretiens qui peuvent être consultés à l'adresse suivante [http://cepel.edu.umontpellier.fr/banques-de-donnees-opera-2]. Le second programme, Programmatic Action in Times of Austerity: Elites Competition in the health sector Governance in the health sector in France, Germany, United Kingdom (England) and the US, dit ProAcTA, 2008-2018 (ANR-17-FRAL0008-01 / DGF BA 1912/3-1) est en cours. Dans ce cadre, nous avons pu réaliser, entre juin 2018 et février 2019, une vingtaine d'entretiens à Washington DC avec certains acteurs-clés de la réforme de l'Affordable Care Act pour tester l'hypothèse développée dans le présent article.
} 
publiques, leurs trajectoires professionnelles durables à l'intérieur des cercles de pouvoir et leur redéfinition du rôle de «gardien de politique ${ }^{5}$ comme promoteur d'une réforme à la fois ambitieuse et acceptable par les intérêts en présence.

\section{Les nouvelles politiques créent-elles de nouvelles élites ?}

Rappelons tout d'abord, le contexte dans lequel ces élites sont amenées à agir en matière de l'assurance maladie. Héritant d'un État fédéral traditionnellement illégitime et aux institutions fragmentées (Steinmo, 2010), l'action publique centralisée y prend, sauf période exceptionnelle, une forme indirecte (Balogh, 2009 ; Mettler, 2011). Cette situation s'est trouvée aggravée par la bipolarisation de la vie politique et un déplacement vers la droite de l'échiquier politique à partir des années 1970 (Pierson, Skocpol, 2007).

En conséquence, les États-Unis ont été plus prompts que les autres démocraties occidentales dans la contestation du pouvoir des élites d'État. Jugées incapables de s'appuyer sur des politiques keynesiennes en déclin, elles ont été tenues, par une partie de la classe politique convertie aux idées néo-libérales, pour responsables de la crise économique et, plus généralement, de la bureaucratisation des sociétés occidentales (Pierson, 1994). De fait, dès les années 1960, sous les présidences Kennedy et Johnson, notamment à l'occasion de la « guerre contre la pauvreté », le rôle des élites d'État avait déjà été limité à certaines politiques urbaines et sociales (Derthick, 1979). Par la suite, la professionnalisation des élites partisanes et la promotion du nouveau management public par les gouvernements conservateurs ont favorisé la remise en question de leur monopole en matière de formulation de politiques (Page, Wright, 1999; Suleiman, 2003). À la fin des années 1970, les personnes nommées politiquement au sommet de l'État ont ainsi pu former un «gouvernement des étrangers » formés d'« oiseaux de passage » (Heclo, 1977) défiant le pouvoir des hauts fonctionnaires dans de nombreux secteurs de politiques.

Le système d'assurance maladie porte, plus encore que dans d'autres domaines de l'action fédérale, les stigmates de cet héritage. Après une tentative initiale pour sa mise en place lors de la Première Guerre mondiale où son idée même fut qualifiée de «complot germanique », le New Deal a été marqué par l'échec d'une National Health Insurance (Oberlander, 2019). Le modèle du « payeur unique » (single payer) - qui ressurgit régulièrement et sans succès depuis à l'occasion de chaque élection présidentielle à la gauche du Parti démocrate - a ainsi été réduit au statut d'utopie inatteignable dans le contexte d'un déroulement normal de la vie politique américaine (Brown, 2019). L' «exceptionnalisme américain » s'est donc exprimé pleinement puisque l'assurance maladie a trouvé comme socle de déploiement un secteur privé complété imparfaitement, au fur et à mesure de saillies de l'État fédéral, par des programmes destinés à couvrir des publics spécifiques dont les emblématiques Medicare (personnes de 65 ans et plus) et Medicaid (personnes à faible revenu) demeurent les fers de lance (Beaussier, 2016). En a résulté un «patchwork » de plus en plus complexe et coûteux à organiser et gérer (Marmor, Oberlander, 2011).

Un changement de logique de gestion de Medicare et Medicaid a été amorcé dès la présidence Carter entraînant une remise en question progressive du profil dominant des élites bureaucratiques et technocratiques en charge de la gestion de ces programmes (Brown, 1983 a et b). La création, en

\footnotetext{
${ }^{5}$ Pour Philip Selznick, les «custodians of policy» sont des individus ayant pour projet la protection de certaines «valeurs sociales » en vue d'affirmer leur leadership et leur autonomie au sein d'une organisation (Selznick, 1957, p. 120). Notre définition est plus extensive dans la mesure où le rôle de gardien consiste en la promotion d'une vision du monde pouvant être traduite en programmes soit sur un mode « défensif » lorsque ces derniers sont appliqués, soit "offensif » lorsqu'ils sont à l'état de programme. Toutefois, comme Selznick, nous considérons que l'orientation commune de l'action des «gardiens des politiques » est susceptible de produire « une identité de groupe » (Selznick, 1957, p. 121) qui renforce leur autonomie (Genieys, 2010 ; Genieys, Joana, 2015).
} 
1977, d'une nouvelle administration financière au cœur du ministère de la Santé ${ }^{6}$, la Health Care Financing Administration (HCFA), supervisant de façon transversale la gestion financière de Medicare et Medicaid, a favorisé l'émergence de nouveaux profils professionnels indexant l'action publique sur une approche plus pragmatique notamment en matière de maîtrise des coûts. Joseph Califano, ministre de la Santé d'alors et principal artisan de la création de la HCFA, justifie en ces termes la rupture avec la vision traditionnelle des politiques sociales au sein du Parti démocrate : «Je voulais prouver que les programmes de la Great Society étaient gérables. Ça c'était la priorité. Ensuite, je voulais faire comprendre aux libéraux que l'on devait avoir de la compétence et de l'efficacité en même temps que de la compassion. Il n'y avait pas de notion d'efficacité au sein de l'establishment libéral, aucune notion de ce que cela signifiait. [...] Je pense que [la HCFA] a accompli quelques progrès en termes de management et d'économies. Je pense surtout qu'elle a aidé le ministère à se concentrer sur les coûts, l'efficacité, à faire comprendre ces choses $»^{7}$. La transformation de la configuration institutionnelle du gouvernement des politiques d'assurance maladie qui a été accentuée par l'affirmation du rôle de l'Office of the Assistant Secretary for Planning and Evaluation (ASPE) au sein du ministère de la Santé dans l'analyse microéconomique et prospective du système de santé renforce l'impact de ce phènomène ${ }^{8}$. Il est accompagné également par le développement des nouveaux instruments de régulation de l'assurance maladie, telles que les Health Maintenance Organizations (HMO) ${ }^{9}$. Ces nouvelles institutions et politiques constituent le terrain sur lequel les anciennes élites sectorielles et de nouvelles élites influencées par la policy analysis s'affrontent (Brown, 1983a, p. 17) ${ }^{10}$.

Cette configuration n'est pas sans rappeller le cas français qui, durant la même période, a vu l'émergence de nouvelles élites des politiques de santé à la direction de la sécurité sociale du ministère de la Santé cherchant à s'émanciper de la tutelle du ministère des Finances (Genieys, 2010, p. 108-113). Ces dernières, au profil de «manager du social », ont réussi collectivement à « apprivoiser » la contrainte financière pour redéfinir - en vue de l'investir - le rôle de gardiens de l'interventionnisme étatique dans le secteur de l'assurance maladie. Certes, alors qu'en France, la transformation de la structure et l'influence de ces élites interviennent dans le cadre d'une relative dépolitisation des programmes des partis de gouvernement, elle doivent, dans le cas américain, composer avec la droitisation de la sphère politique. Néanmoins, dans les deux cas il y a constitution d'un groupe d'élites sectorielles difficilement contournable lorsqu'il s'agit de redéfinir l'action étatique en matière d'assurance maladie.

Pour appliquer cette hypothèse au cas américain, on partira des travaux d'Hugh Heclo (i) tout d'abord, en considérant la bureaucratie washingtonienne à la structure duale (formée de personnes nommées politiquement et d'élites administratives) comme correspondant peu ou prou à celle où évoluent les élites d'État européennes (Heclo, 1984, p. 12-13); (ii) ensuite, en postulant que les

\footnotetext{
${ }^{6}$ Department of Health, Education and Welfare (DHEW) devenu en 1979 Department of Health and Human Service (DHHS).

${ }_{7}$ «Interview with Joseph Califano in his office in new york city on 31 august 1995 », by Edward Berkowitz, The HCFA Oral History [https://www.ssa.gov/history/CALIFANO2.html].

${ }^{8}$ L'Office of the Assistant Secretary for Planning and Evaluation a été créé en 1966 au sein du ministère de la Santé. Il est composé à l'origine d'une vingtaine d'analystes développant des recherches prospectives à dominante économétrique pour mesurer l'impact fiscal des «options politiques » (policy options). Aujourd'hui, l'ASPE qui est composé de plus de cent agents, est le véritable bras armé du ministère de la Santé (Genieys, 2020).

${ }^{9}$ Pour Victor Rodwin le HMO « a pour mission d'assurer leurs adhérents et de leur fournir des services médicaux (y compris l'hospitalisation). Ceux-ci adhèrent [à la] HMO par le paiement d'une cotisation forfaitaire qui leur garantit l'accès à une gamme de services médicaux, comprenant souvent des services de dépistage et de médecine préventive » (Rodwin, 1985).

${ }^{10}$ Pour Larry Brown, ces réformes administratives ouvrent l'ère du «corporatisme technocratique » (technocratic corporatism) marquée par des formes renouvelées d'expertise et d'intrumentation des politiques publiques ainsi qu'une redéfinition des relations avec des groupes d'intérêts comme l'American Medical Association ou encore l'American Hospital Association (Brown, 1985).
} 
«politiques en tant qu'activité washingtonienne et intra-muros» sont produites par l'action conjointe du Congrès et du pouvoir exécutif (Heclo, 1978, p. 98). Ainsi, on considère que les individus qui ont intégré, sur la longue durée, ce périmètre du pouvoir et de son action, sont susceptibles de constituer, dans certains secteurs de politiques, des groupes d'élites professionnalisées dans les affaires de gouvernement. Fort de ces trajectoires sectorielles durables, ces élites des politiques se trouvent en position d'influer sur le processus de prise de décision et d'endosser le rôle de gardien d'une vision programmatique ayant pour finalité le renforcement de la capacité régulatrice de l'État.

L'analyse longitudinale des trajectoires professionnelles des élites des politiques de santé, au cours de la période allant de l'échec du Plan Clinton au succès de la réforme Obama (Patient Protection and Affordable Care Act, permet d'observer comment certaines d'entre elles ont rompu avec l'héritage politique des « réformateurs » de la Social Security Administration (SSA), arc-boutés sur la défense des programmes Medicare et Medicaid (Oberlander, 2003). Elles se sont emparées, par la même occasion, du rôle de «gardien » de la politique d'assurance maladie, dont ces derniers avaient la charge, pour moderniser un système, inéquitable et coûteux, quasiment au point mort depuis les années 1960 (Beaussier, 2012).

\section{Une formation initiale et continue à la Health Policy Analysis}

À partir de la base de données OPERA, nous avons réalisé une analyse sociographique des personnes ayant occupé des postes de pouvoir durant plus de six années en tant que staffer du Congrès et/ou nommé politiquement à la Maison Blanche ou au ministère de la Santé. Ces postes ont été retenus en raison de leur lien potentiel avec le processus de prise de décision en matière d'assurance maladie. L'étude des élites de la santé occupant les pouvoirs exécutif et législatif a permis de mettre en avant : la spécificité de la nature de leur formation; les universités fréquentées ; leur trajectoire professionnelle en direction des sommets de l'État; la durée moyenne de leur carrière; les transferts, détachements, promotions dont ils ont été l'objet; la typologie de leurs carrières institutionnelles.

Outre une relative féminisation de la population, le curriculum de ces élites confirme qu'elles possèdent un haut niveau de formation (Darviche et al., 2013). L'analyse du type d'études permet de relever un nouveau phénomène: leur spécialisation dans les Schools of Public Health d'universités se situant, pour la plupart, dans la région de Washington D.C. Ce changement fait suite à la création de Schools of Public Policy par un progamme de la Fondation Ford (1975) ${ }^{11}$. Douze mastères, inspirés par une approche en termes de «Policy Science » centrée sur la formation aux politiques publiques et à leur évaluation, ont ainsi été mis en place (Allison, 2006). Ces institutions qui proposent des formations interdisciplinaires permettent à leurs étudiants de saisir les enjeux qu'implique l'évaluation des politiques publiques et les dotent de diplômes universitaires spécifiques (Mastère en politique publique, Mastère en santé publique et même doctorat) nécesssaires à la future spécialisation sectorielle au Congrès et/ou au sein de l'exécutif (Darviche $e t$ al., 2013, p. 15-18).

La création de ces écoles correspondait à la volonté politique de former, dans une perspective moins bureaucratique, la nouvelle génération d'experts et de techniciens amenée à gouverner les politiques publiques. La devise informelle portée par la nouvelle génération de démocrates libéraux était «to dream up ways to make the world a better place ». L'enseignement qui intègre les questions de qualité de vie et d'équité sociale dans un horizon structuré par la nécessaire prise en compte de l'«efficience» de l'action gouvernementale, a contribué à la formation de ces nouveaux

\footnotetext{
${ }^{11}$ À l'origine du programme de la Fondation Ford on dénombre 8 écoles. Elles sont, aujourd'hui, environ quarante et forment plus de 10000 diplômés par an. Cf., Pierson, J., Schaefer, N., (2013), «The Problem with Public Policy Schools », The Washington Post, 6 décembre.
} 
« concepteurs de politiques publiques »(policymakers). Depuis les années 1980, ces derniers ont progressivement investi les postes-clés en matière de santé au sommet de l'État (Encadré 2). Les trajectoires professionnelles des élites santé confirment que la maîtrise d'un tel savoir sur les politiques de santé s'est imposée comme une ressource majeure en vue du développement de carrrières durables aux sommets du pouvoir dans ce secteur (Encadrés 2 et 4).

Cette washingtonisation des cursus universitaires élitaires tranche avec le cas français où le binôme Sciences Po Paris-École nationale d'administration reste dominant dans la formation des hauts fonctionnaires gouvernant les politiques de santé. En effet, si d'une certaine manière, les Schools of Public Policy ou School of Public Health jouent un rôle équivalent à celui de l'ENA dans la formation de personnels habilités à gouverner les politiques, elles s'en distinguent, d'abord parce qu'elles ne permettent pas d'accéder directement au statut de haut fonctionnaire de carrière comme en France et, ensuite, parce qu'elles garantissent l'acquisition d'un savoir-faire spécialisé en matière de politique publique, en général, et de politique de santé, en particulier. Il a fallu attendre les années 1990 pour que l'évaluation des politiques intègre la formation des fonctionnaires et 2015 pour que Sciences Po Paris ouvre une «École d'affaires publiques » (Hassenteufel, Le Galès, 2018, p. 317). Toutefois, aux États-Unis, l'acquisition «précoce» de compétences en matière de politiques publiques ne se limite pas à une formation universitaire initiale, mais se poursuit au cours de longues carrières au sein ou en dehors de l'État fédéral.

\section{Accumulation et transmission de savoir-faire sur les politiques de santé}

L'analyse sur la longue durée des étapes successives des trajectoires professionnelles de ces élites à Washington D.C. indique que l'exercice des fonctions dans le secteur public comme dans le secteur privé est toujours en lien avec les politiques de santé. Ce qui permet à ces élites d'accumuler du savoir-faire pour l'investir, le moment venu, dans le gouvernement des politiques. Il s'agit, indéniablement, d'une caractéristique dont les élites bureaucratiques de la santé des générations précédentes étaient dépourvues.

Les « réformateurs » avaient plutôt une culture bureaucratique liée à la mise en œuvre et la gestion des programmes Medicare et Medicaid (Brown, 1983 ; Oberlander, 2003). Pour la génération suivante des élites des politiques de santé intégrant les coulisses du pouvoir dans les années 1990, le passage par les nouvelles filières académiques s'impose, de plus en plus, comme une obligation pour accéder à un premier poste de staffer personnel d'élus du Congrès impliqués dans les commissions parlementaires en charge des questions de santé (Encadré 2). L'analyse de leurs trajectoires professionnelles permet, par ailleurs, de souligner l'importance de ce facteur dans le développement de carrières durables au sommet du pouvoir (Genieys, 2020, chapitres $3 \& 4$ ).

Si la policy analysis est un élément important de la formation des élites des politiques de santé de sensiblité politique démocrate, ces dernières peuvent, grâce au système du revolving door, et lorsqu'un changement de majorité politique intervient, choisir un poste de chercheur ou d'enseignant associé en santé publique dans le monde académique. Ainsi, nombre de staffers et la grande majorité des nommés politiquement titulaires d'un doctorat ont partagé leur connaissance pratique de l'action publique dans l'une ou plusieurs des nombreuses écoles de politiques publiques ou de santé publique de Washington DC telles que : le Center for Health Policy Studies de la School of Medecine de Georgetown University, le Department of Health Policy and Management de John Hopkins University, la School of Public Health de la George Washington University tout comme celles de l'American University ou encore de la George Mason University. Ces institutions sont devenues des lieux privilégiés de reconversion, transitoire ou définitive, pour des élites de la santé, essentiellement de sensibilité démocrate. Ces départements académiques se montrent très intéressés par des expériences pouvant être mises au service de formations professionalisantes comme les Mastères en santé publique (Master of Public Health-M.P.H.). 
Ainsi, des anciens de l'époque Clinton mais aussi certains de ceux entrés dans la carrière au Congrès durant la Présidence de G. W. Bush, ont effectué un passage à la School of Public Health de l'Université John Hopkins à Baltimore (Diane Rowland et Tom Morford, Cibèle Bjorklund, Liz Fowler), au Center for Health Policy Studies de l'École de Médecine de l'université de Georgetown (Judy Feder, Brian Biles, Wendell Primus, Karen Pollitz, Jeanne Lambrew) ou encore à la School of Public Health and Health Services de l'Université George Washington (Andy Schneider, Ruth Katz, Carolyn Clancy). Cette porosité professionnelle entre la sphère de gouvernement des politiques et le milieu académique constitue un facteur de différenciation des trajectoires professionnelles des élites démocrates de celles des élites républicaines de la santé. Ces dernières sont plutôt liées au monde économique et enclines à monnayer leur savoir-faire auprès des puissants lobbies du secteur. On peut évoquer ici, à titre d'exemples, les cas David Abernethy qui est devenu vice-président de la compagnie Health Insurance Plan Administrators, et de «Chip » Khan, président de la Federation of American Hospitals. Le cas de Joe Antos, ancien visiting professor à la Gilling School of Global Public Health à l'Université de la Caroline du Nord et plus tard Wilson H. Taylor Scholar en Santé à l'American Enterprise Institute est l'exception qui confirme la règle du côté républicain.

L'analyse des trajectoires professionnelles confirme que l'accroissement continu d'un savoir-faire sur les enjeux de politiques, institutionnels et sociaux de la santé tout au long d'une carrière constitue un important marqueur identitaire des élites washingtoniennes de la santé. Les allersretours entre une expérience dans les coulisses du pouvoir fédéral et celles de l'enseignement et de la recherche académique poussent ce type singulier d'élites des politiques publiques à s'investir naturellement dans la définition d'orientations programmatiques en matière de santé au sein des puissants think tanks washingtoniens.

\section{Une circulation sur la longue durée à l'intérieur et autour de l'État}

Le type et la durée de la spécialisation dans un secteur spécifique sont aussi des marqueurs distinctifs des trajectoires professionnelles étudiées (Encadré 4). La durée moyenne des carrières dans le secteur de la santé est ainsi supérieure à vingt ans (Darviche et al., 2013). Ces carrières se distinguent par une forte mobilité mais à l'intérieur de ce secteur (ibid., 2013, p. 22-26). Cette sectorisation des carrières est un élément important de la construction de l'action étatique. En effet, même si aux États-Unis, il est commun d'observer de fréquents allers-retours entre sphères publiques et privées, ces mouvements, dans la mesure où ils restent circonscrits au sein d'un même secteur d'action, tendent à favoriser l'émergence de réseaux d'interconnaissance qui vont au-delà des frontières formelles de l'État (Lepont, 2014, 2016a).

À côté des élites démocrates de la santé qui ont opté pour un repli professionnel transitoire à l'Université, d'autres se reconvertissent momentanément à Washington D.C. dans le secteur privé, essentiellement dans les think tanks (Brookings, Urban Institute, Center on Budget and Policy Priorities), fondations (Robert Wood Johnson Foundation, Henry J. Kaiser Family Foundation) et associations à but non lucratif mais, plus rarement, dans les affaires ou les groupes d'intérêts (Encadré 4). Toutefois, qu'elle soit acquise à l'intérieur ou à l'extérieur des frontières institutionnelles de l'État, leur expertise des politiques publiques tend à renforcer les capacités régulatrices du secteur public. Un de nos interviewés, ex-staffer au Congrès, précise que travailler dans une institution à but non lucratif constitue une caractéristique commune à la «famille des collaborateurs du Representative Henry Waxman ». «Personne, poursuit-il, ne quitte [vraiment] le staff Waxman. Quand on se fait virer, on reste amis, on s'appelle. Un peu comme un réseau. [...] Certains commentateurs politiques de droite [sont allés jusqu'à dire] qu'Henry contrôlait une mafia libérale des politiques publiques. [...] Il est intelligent, attentionné, on travaille aisément avec lui et nous lui sommes tous très dévoués. [...] Nous restons là, année après année... Dans le livre consacré au Congrès, les gens s'étonnent de la longévité du staff de M. Waxman : un bon staff, [composé] de gens brillants qui savent ce qu'ils font et qui sont là depuis toujours. Il engage aussi 
des jeunes gens car certains d'entre nous allons enseigner dans des facultés de droit, d'autres vont représenter des organisations à but non lucratif ${ }^{12}$.

Malgré leurs différences quant au choix de leur activité professionnelle dans le secteur privé (plutôt à but lucratif pour les républicains versus plutôt à but non lucratif pour les démocrates), ces élites se sont investies dans les années 2000, durant le deuxième mandat de G. W. Bush, dans des think tanks bipartisans «inside-the-Beltway ( (Lepont, 2014, p. 393-468) pour débattre d'un projet de réforme de grande envergure de l'assurance maladie (Genieys, 2020, chapitre 8). De fait, à côté des think tanks traditionnels, The Urban Institute, the Brookings Institution ou encore le Center on Budget and Policy Priorities (1981), côté démocrate ${ }^{13}$, et l'American Entreprise Institute et la Heritage Foundation, côté républicain, de nouveaux think tanks bipartisans vont apparaître - le Center for American Progress (2003), le Bipartisan Policy Center [for Health Reform] (2007), et le Hamilton Project (2006), le Health Policy Consensus Group (2003) - en vue d'identifier les points de convergence. Le Commonwealth Fund, la Kaiser Family Foundation et la Robert Wood Johnson Foundation qui (ré)active l'Alliance for Health Reform, pour en faire un forum de médiatisation au sein duquel d'anciens démocrates de l'administration Clinton mais également certains de leurs opposants républicains d'alors, vont développer les contours d'une vision bipartisane du projet de réforme en intégrant notamment les notions de marché et de contrôle des coûts.

L'analyse des trajectoires professionnelles des élites de la santé montre ainsi que la plupart des individus occupant les postes dans les coulisses du pouvoir ont, durant leur longue carrière, accumulé du savoir-faire sur les politiques publiques en alternant périodes d'action gouvernementale et périodes de participation à une réflexion prospective sur le devenir du système. Elle permet d'identifier trois ideaux type de circulation de ces élites à l'intérieur du pouvoir et au sein de sa proche périphérie (Encadré 3). Le premier, celui des migrants institutionnels, correspond à ceux qui ont œuvré, durant leur carrière, aussi bien au sein du pouvoir exécutif que législatif. Leur circulation s'effectue bien souvent dans le contexte de l'iniation d'une réforme dans le but de fluidifier les relations entre l'administration du président et le Congès. Le second, celui des technocrates facilitateurs, renvoie à ceux qui circulent uniquement à l'intérieur des deux pouvoirs et qui y occupent progressivement les postes les plus élévés. Ils sont dotés d'une forte capacité de transformations des orientations programmatiques en textes de loi. Enfin, le troisième, celui des bureaucrates des politiques, caractérise des individus bénéficiant d'un statut de fonctionnaire au sein d'un des deux pouvoirs. Ils sont dotés d'un fort savoir-faire technique (statistiques, comptabilité, droit, etc.) pour le contrôle des politiques.

Précisons que cette spécialisation durable et washingtonienne est beaucoup plus prégnante chez les démocrates. De fait, ce groupe professionnel constitue un vivier d'élites de la santé au sein duquel les leaders politiques sont amenés à puiser pour composer leur entourage politique lors d'un changement de majorité politique au Congrès ou encore à l'arrivée d'un nouveau président à la Maison Blanche. À l'instar du cas français, où les élites de la santé circulent lors des changements de majorité politique à l'intérieur de l'État, notamment au sein des corps d'inspection (Inspection générale des affaires sociales) ou encore des grands corps (tels que la Cour des comptes), leurs homologues américains circulent à Washington dans les nombreuses organisations sectorielles privées non lucratives (Encadré 4).

C'est dans ce contexte que les élites des politiques de santé accédant au sommet du pouvoir sectoriel ont notamment mobilisé l'argumentaire des coûts contenus. Forts dans l'art de la persuasion et l'argumentation, maîtrisant l'analyse du contexte politique, la grammaire des

\footnotetext{
12 OPERA Interview, Washington D.C., may 28, 2010: 7 \& 8 [http://cepel.edu.umontpellier.fr/files/2015/04/ANROPERA-Entretien_sante_45.pdf].

${ }^{13}$ Précisons que l'Urban Institute et la Brookings Institution préfèrent se classer comme bipartisans. Mais, en réalité, les républicains ont très peu recours à ces deux think tanks.
} 
politiques publiques et la micro-économie, ces élites jouent un rôle déterminant dans la fabrication des orientations programmatiques (Genieys, Hassenteufel, 2012). Leurs longues trajectoires professionnelles les pourvoient d'une autorité politique leur permettant de concevoir, préparer et chiffrer des politiques pouvant être défendues face aux assauts de la droite conservatrice, acceptées par les démocrates centristes et opposables à l'opinion publique.

\section{La définition des contours d'une réforme politiquement acceptable}

Aux États-Unis, toute réforme de l'assurance maladie doit réussir plusieurs épreuves, ce dont le modèle du «payeur unique » (Encadré 1) a été jusqu'à ce jour incapable. Outre l'émiettement institutionnel et la forte bipolarisation et droitisation politiques évoqués plus haut, une telle réforme doit tenir compte des opérateurs privés à l'important potentiel de lobbying durablement installés au sein du système assurantiel (Epperson, 2019; Oberlander, 2019, p. 1498-99) mais surtout d'une opinion publique pas forcément insensible à l'argument du "péril socialiste» et celui de l' «augmentation des impôts » et qui demeure majoritairement attaché au système d'assurance privée fournie par les employeurs (Brown, 2019 ${ }^{14}$ ). Ainsi, plus de la moitié des Américains interrogés, peu au fait des conséquences d'une telle réforme, pensent qu'elle ne remettrait pas en question leurs actuelles polices d'assurance privées (ibid., p. 1508). Alice Rivlin, à la très longue carrière dans le secteur de la santé notamment à des postes importants au sommet de l'État fédéral dont celui de directrice adjointe de l'Office of management and Budget à la Maison Blanche -, précise qu'au moment de la tentative de réforme Clinton les citoyens américains «ne voulaient pas aller vers un système de type européen, mais souhaitaient garder leur "employer-based insurance" "15. En effet, surtout à partir des années 1980, l'approche en termes de "payeur unique » a acquis aux États-Unis le statut de «réforme (peut-être) admirable mais impossible politiquement »(Brown, 2019, p. 1509).

Quinze années plus tard, sous la présidence Obama, les données n'ayant donc pas fondamentalement changé, améliorer le système d'assurance maladie (augmenter le nombre d'assurés) impliquait pour ses promoteurs de mener la réforme sur une base pragmatique, d'une part, en reprenant les fondements du système existant (maintien des institutions et acteurs existants, publics comme privés), d'autre part, en associant le Congrès à son élaboration, évitant ainsi sa mise à l'écart fatale de 1993 (Beaussier, 2016, p. 89 sq.). Dès le départ, la réussite de la réforme passait donc pour le président Obama et son équipe par une étroite collaboration de l'exécutif avec le Congrès. Ce dernier va ainsi entamer le travail législatif sur la base d'une feuille de route générale (briefing memo) rédigée par Nancy-Ann DeParle ${ }^{16}$ - vétéran de l'administration Clinton ${ }^{17}$ - chargée de piloter le processus de prise de décision.

La réforme s'est donc orientée vers une approche non disruptive. Dans cette perspective, la question du contrôle des coûts qui se trouve régulièrement au devant de la scène et qui fait l'unanimité auprès des acteurs publics comme privés du secteur, n'opère pas dans l'absolu ; elle ne peut se déployer que dans ce cadre politique spécifique et très contraint. Ce «provincialisme américain »

\footnotetext{
${ }^{14}$ S'appuyant sur un sondage Kaiser's Family Foundation de janvier 2019 (question 6 [https://www.kff.org/healthreform/poll-finding/kff-health-tracking-poll-january-2019/]), Larry Brown (2019, p. 1508) indique que, même de nos jours, l'enthousiasme du public pour le modèle du «payeur unique » se nuance fortement dès qu'il apprend que cela éliminerait les assurances privées, impliquerait une augmentation des impôts, une remise en question de Medicare tel qu'il existe et une possible augmentation des délais pour les analyses et les traitements.

${ }^{15}$ Entretien Santé OPERA - Washington DC : n³4, 28 avril 2010, p. 4.

${ }^{16}$ [http://www.documentcloud.org/documents/328161-health-care-memo.html].

${ }^{17}$ De Parle comme Jeanne Lambrew, autre grande figure de la réforme côté pouvoir exécutif (cf. infra), correspondent au type du «technocrate facilitateur» (Encadré 3). Elle ont fait, toutes deux, leur carrière publique fédérale exclusivement au sein de l'exécutif (Maison Blanche et ministère de la Santé). Pour une analyse détaillée de leurs trajectoires porfesionnelles, et notamment du fait qu'elles occupent à tour de rôle les mêmes fonctions dans les sommets du pouvoir exécutif se reporter aux chapitres 4 et 5 de Genieys (2020).
} 
(Marmor, Oberlander, 2011, p. 128) a impliqué qu'au regard du tabou d'une assurance-maladie publique, le débat se situe sur le terrain de l'acceptabilité ou non d'une offre publique d'assurance («public option », ci-après «option publique » [Encadré 1]) pouvant être proposée en même temps que les assurances privées.

Rappelons que plusieurs organismes publics sont en charge du contrôle du coût des politiques fédérales. En se focalisant sur les trajectoires professionnelles des élites de la santé, on a pu constater que nombre d'entre elles ont fréquenté ces organismes (Encadré 4$)^{18}$. Ces élites se sont formées à l'évaluation et la gestion du coût des politiques de couverture maladie et ont pu maximiser leur influence sur le processus de prise de décision. Leur apprentissage s'est réalisé dans l'exercice de fonctions de Program Associate Director au sein de l'Office of Management Budget $(\mathrm{OMB})$ à la Maison Blanche ${ }^{19}$ ou encore en occupant un poste au sein de la direction financière (HCFA renommée $\mathrm{CMS}^{20}$ depuis 2001) et l'Office of the Actuaries au ministère de la Santé. Du côté du Congrès, outre le Congressional of Budget Office (CBO), l'agence dédiée à l'évaluation prospective des dépenses, les staffers de carrière spécialisés dans les politiques de santé ont pu se former de manière comparable au sein des puissantes commissions financières du Sénat et de la Chambre des représentants en se confrontant quotidiennement à la conception mais aussi au chiffrage des politiques (commissions Finance and Budget au Sénat et Ways and Means à la Chambre des représentants). À côté des institutions exerçant le contrôle financier sur les politiques d'assurance maladie, l'occupation de la fonction de Deputy Assistant Secretary for Planning and Evaluation (ASPE) joue un rôle important dans le processus d'apprentissage professionnel de la nouvelle élite des politiques de santé ${ }^{21}$. Un nombre important de ceux qui ont pesé sur le choix des orientations programmatiques dans les politiques de santé ont occupé cette fonction (Encadré 4).

Le passage par ces institutions a été un élément déterminant dans la construction d'une vision commune quant au renouveau du rôle de gardien des politiques d'assurance maladie. C'est ainsi que des anciens de l'administration Clinton - tels que Bruce Vladeck, Nancy-Ann Deparle et Jeanne Lambrew - qui ont compris le rôle déterminant joué par le CBO dans l'échec de la réforme Clinton, se sont succédé à d'importants postes à responsabilité au sein de la HCFA ou l'OMB Au Congrès, ces « anciens », bientôt rejoints par des nouveaux entrants, tels que Liz Fowler, Cibele Bjorklund ou encore Lisa Konwinski, ont occupé des positions-clés au sein des commissions "financières ». Fortes de cette expérience, ces élites se sont mobilisées collectivement, dans les années 2000, au sein de think tanks bipartisans (Alliance for Health care Reform, Center on Budget and Policy Priorities, Hamilton project) pour que cet enjeu trouve sa place dans le programme de réforme en cours d'élaboration. L'arrivée des économistes Peter Orzag et Jason Furman, codirecteurs du Hamilton project, va appuyer cette orientation des politiques de santé habituellement peu prisée parmi les experts démocrates. Dès sa nomination par les démocrates à la direction du CBO, Orzag va travailler dans une triple perspective : amélioration de la justice sociale, maitrise de la trajectoire du budget santé et réforme du système d'assurance maladie. De son côté, Jason Furman intégrera le Council of Economic Advisors du président Obama pour huit années (Genieys, 2020, chapitre 8).

À ce titre, l'analyse de nos entretiens révèle que le contrôle des coûts est loin d'être un dispositif

\footnotetext{
${ }^{18}$ Précisons que la majorité de ces institutions, souvent constituées sous la forme d'agences, ont été créées sous l'administration Johnson suite à la mise en place du Planning Programming Budget System (PPBS).

${ }^{19}$ L'OMB a remplacé le Bureau of the Budget sous la présidence Nixon en 1970 avec des responsabilités et une capacité d'expertise accrues. Dans le contexte de conflit avec le Président Nixon (en pleine affaire du Watergate) et, plus généralement, afin d'éviter une monopolisation de l'expertise budgétaire par le pouvoir exécutif et garder le contrôle de l'exécution du budget fédéral, le Congrès, contrôlé par la majorité démocrate, vote, en 1974, la création d'une agence fédérale non partisane en son sein : le Congressional budget office (CBO). Alice Rivlin et Peter Orzag qui ont joué un rôle déterminant dans la préparation de l'ACA, ont occupé la direction de ces deux institutions (Joyce, 2011, p. 186-87).

${ }^{20}$ Center for Medicaire and Medicaid Services.

${ }^{21}$ Depuis la présidence Clinton, l'ASPE (cf. note 9) s'est imposée à la fois dans l'élaboration du « HHS Strategic Plan » et comme boîte à idées pour la réforme du système de santé.
} 
discursif pouvant être (re)formulé à loisir pour soutenir telle ou telle réforme mais une prétention dont la preuve de réalité doit être apportée ${ }^{22}$. Les batailles politiques menées lors de l'élaboration de l'ACA (2008-2010) feront de l'alignement derrière l'argumentaire des coûts contenus un élément important d'identification et d'autonomie d'action de ces insiders des politiques de santé par rapport à des acteurs démocrates défenseurs de l'«option publique » mais dépourvus de l'expérience quotidienne du pouvoir. D'ailleurs, ce groupe d'élites a été porteur d'une stratégie de réforme comportant quatre volets : (i) la nécessité d'intégrer dans le nouveau dispositif les éléments du système existant, (ii) la mobilisation des forces du marché, (iii) le contrôle des coûts et (iv) l'obtention d'un consensus politique le plus large possible (Genieys, 2020).

\section{L'Affordable Care Act : l'affirmation du rôle de gardien des politiques de santé}

L'analyse des trajectoires professionnelles des « initiés » révèle la présence de ceux qui ont circulé dans les coulisses du pouvoir washingtonien avant le démarrage du processus de réforme par le président Obama (Genieys, 2020, chapitre 9). Cette circulation, tout particulièrement celle de certains d'entre eux au moment du lancement de la réforme entre exécutif et législatif, est révélatrice de leur stratégie collective d'investissement des postes-clés durant la réforme (Encadré 5). Notre recherche révèle que cette lutte entre élites insiders et outsiders des sommets de l'État fédéral intervient dans un contexte plus global qui est celui de la capacité de ce dernier à reconfigurer ce secteur en élargissant son champ d'action. Les gardiens des politiques de santé qui ont émergé à partir de l'administration Clinton autour d'un nouveau projet de réforme avaient pour objectif d'étendre ce contrôle sur un système d'assurance maladie qui n'avait pas connu d'avancées majeures depuis les années 1960. Ils ont lancé un projet initialement fragmenté pour progressivement combiner public et privé en vue d'assurer au mieux l'impératif du contrôle des coûts, dans un contexte politique excluant très majoritairement (classe politique comme opinion publique) un système public comparable à celui des autres démocraties occidentales.

Si cette proposition - qui est finalement devenue celle du Sénat au détriment de l'«option publique ${ }^{23}-1$ 'a emporté, c'est aussi parce qu'elle a passé la redoutable épreuve des estimations du CBO. L'évaluation prospective opérée par ce dernier, a estimé que le coût pour les finances publiques sur dix ans du préprojet, basé sur l'«option publique», soutenu par la Chambre (l'Affordable Health Care for America Act) était nettement supérieur que celui avancé par le Sénat (Affordable Health Choice Act) (1 052 milliards de dollars contre 870) (Beaussier, 2012). De fait, le «mieux-disant» de ce dernier a été un élément décisif dans la forme finale prise par la réforme Obama (Hacker, 2010, p. 870). Comme l'indique un adversaire de « l'option publique » qui a joué un rôle important dans la réforme au sein du Congrès et du pouvoir exécutif, "non seulement notre projet de loi était la moins chère [...], mais en plus c'est le seul qui dès le projet initial a réellement baissé les coûts [...]. Bon! il a fini par être le projet du Sénat ${ }^{24}$. Pour les nouveaux gardiens des politiques de santé, il est établi que toute action publique pensée et planifiée économiquement sur le long terme et apporte la preuve qu'elle constitue, par rapport à des programmes concurrents, est l'option la moins coûteuse.

L'affirmation du rôle de gardiens des politique de santé se retrouve dans la prise de distance par rapport à des experts extérieurs à leur univers. Ainsi lorsqu'on les interroge, par exemple, sur l'influence supposée de l'universitaire Jacob Hacker, promoteur de l'«option publique », au moment de la réforme, l'un de ces insiders précise : «C'est juste un universitaire. Il a été consulté

\footnotetext{
${ }^{22}$ Entretien OPERA, Washington D.C., 30 avril 2010 ; Entretien ProAcTA, Washington D.C., 18 juin 2018 ; Entretien ProAcTA, Washington D.C., 19 juin 2018 ; Entretien ProAcTA, Washington D.C., 27 mars 2019.

${ }^{23}$ Entretien ProAcTA, Washington D.C., 18 juin 2018. La «public (health insurance) option » était initialement prévue dans la réforme Obama. Elle ne remettait pas en cause les assurances (privées) existantes mais proposait une alternative publique.

${ }^{24}$ Entretien ProAcTA, Washington D.C., 18 juin 2018.
} 
par la Maison Blanche mais n'y a jamais été nommé » ${ }^{25}$. Un autre poursuit en indiquant que «[les universitaires] n'ont pas d'influence sur notre travail direct [...] on les écoute sur ce qu'ils font quand on a le temps ${ }^{26}$. Selon un autre conseiller en politique de santé qui a joué un rôle important à la Maison Blanche sous Clinton et Obama, «les fondations politiques pour une bonne réforme » incluent toujours quatre «pieds»: une réforme de l'assurance qui élargit l'accès, instaure l'obligation d'être assuré qui intègre les individus au système, propose des aides permettant un accès abordable au marché de l'assurance, et contrôle des coûts. Sur le caractère non négociable de ce dernier il précise que "le quatrième et dernier pied de la chaise est la réforme du système financier lui-même. Les coûts explosent, alors vous devez mettre en place des mécanismes de contrôle, vous devez mettre en place des mécanismes d'amélioration du rapport qualité-prix (value purchasing) ou de besoin de financement, afin d'assurer la durabilité du système de santé ${ }^{27}$. Partant de là, on voit bien pourquoi ces insiders dotés d'une longue experience du pouvoir, et pour certains d'entre eux marqués par leur échec sous la présidence Clinton, s'opposent radicalement à toute tentative d'influence des « oiseaux de passage » (Heclo, 1977).

La victoire de l'orientation programmatique défendue par les gardiens des politiques de santé tient à leur capacité de transformer l'expérience des échecs passés en ressource politique. En effet, la recherche d'une réforme au contenu consensuel a conduit à l'élimination d'alternatives plus clivantes comme l' «option publique » (McDonough, 2012). À cette occasion, certains auteurs, à l'image de Steven Brill, ont mis à l'index un supposé travail de promotion des intérêts des lobbies sectoriels au sommet du pouvoir par des personnalités en charge de la réforme, comme Liz Fowler ou encore Jeanne Lambrew (Brill, 2015). Rappelons, tout d'abord, que les groupes d'intérêts privés étaient alors très divisés pour espérer contruire un front uni pouvant imposer leur vue au réformateur (Mizruchi, 2013). Mais plus spécifiquement lorsque l'on étudie la trajectoire professionnelle de Fowler et Lambrew on constate que, l'une comme l'autre, ont passé beaucoup plus de temps à gouverner les politiques de santé que travailler pour le secteur privé. Liz Fowler a été en charge des questions de santé à la commission des Finances du Sénat de 1999 à 2005, avant de rejoindre les entreprises privées Health Policy alternative puis WellPoint Inc. Elle est revenue au Sénat dans la même commission en 2008 puis a intégré, en 2010, le ministère de la Santé comme deputy director for policy et est devenue dans la foulée, de 2011 à 2012, assistante spéciale en matière de santé du président Obama. De son côté, Jeanne Lambrew est passée par l'OMB durant le clintonisme tardif avant d'effectuer un passage à l'Université d'Austin. Elle a occupé ensuite, sous Obama, le poste de policy director dédié au suivi de la réforme (Office Consumer Info. \& Ins. Oversight, $O C I I O)$ au ministère de la Santé, puis celui de conseillère spéciale du président pour les questions de santé au National Economic Council de la Maison Blanche (2015). On remarquera au passage, sur la base de ces deux trajectoires, l'importance de leur socialisation professionnelle au sein d'instances de contrôle financier (Encadré 3).

L'analyse croisée des trajectoires professionnelles et de l'orientation programmatique formulée par ce nouveau type d'élites des politiques de santé montre qu'il s'agissait pour elles d'aboutir, coûte que coûte, à une réforme de grande envergure tout en laissant la porte ouverte à sa possible amélioration dans le futur. L'une d'entre elles résume ainsi son expérience : «Une autre leçon que j'ai apprise durant ma carrière est que nous sommes dans la politique et non dans un environnement académique. Aussi, pour mettre en place une réforme, vous devez faire des arbitrages, vous devez faire des compromis avec des choses que vous n'aimez pas. Dans la réforme, certaines dispositions sont moches, je pense que certaines sont même vraiment stupides, de la mauvaise politique publique... Mais au moins, nous l'avons fait, et on peut [toujours] réformer la

\footnotetext{
${ }^{25}$ Entretien OPERA, Washington DC : $\mathrm{n}^{\circ} 45,28$ mai 2010.

${ }^{26}$ Entretien OPERA - Washington DC : $\mathrm{n}^{\circ} 37,1^{\mathrm{er}}$ avril 2010.

${ }^{27}$ Entretien OPERA, Washington DC : 30 avril 2010.
} 
réforme $\gg .{ }^{28} \mathrm{C}$ 'est à ce titre, que les anciens de l'administration Clinton ont eu beau jeu de rappeler que la recherche de la réforme parfaite avait conduit à un échec retentissant laissant, pour longtemps, des millions de citoyens américains sans assurance maladie ${ }^{29}$. Outre le poids du passé et la peur d'un nouvel échec, ces élites ont appris à gouverner les politiques «de l'intérieur » en se prémunissant contre l'influence d' «étrangers ». C'est pourquoi elles se sont attelées à aplanir les conflits entre pouvoirs exécutif et législatif qui étaient à l'origine de l'échec du Plan Clinton. Le travail réalisé discrètement a notamment permis de dépasser progressivement les divergences entre les deux chambres et surmonter l'absence des républicains initialement impliqués dans une projection bipartisane de la réforme (Beaussier, 2012).

Durant toute la période de débat sur la réforme, ces élites ont travaillé pour faire aboutir la dernière version de la réforme élaborée par le Sénat et ce, malgré la perte de la majorité qualifiée suite au décès de Ted Kennedy. Par ailleurs, en raison de leur parcours professionnel, elles ont rassuré le CBO et l'aile droite du parti démocrate sur leur capacité à s'engager sur la voie d'une réforme de l'assurance maladie au coût maîtrisé. C'est en faisant aboutir leur approche spécifique de la réforme - une réforme de "grande envergure » (far reaching reform) contre une réforme systémique (comprehensive reform) qu'elles considéraient comme impossible au regard de leur expérience qu'elles ont pu endosser le rôle de gardiens légitimes des politiques d'assurance maladie.

Avec le recul, on constate que cette approche a abouti à une réforme qui n'a, jusqu'à présent, pas pu être démantelée et ce, en dépit des efforts répétés de l'administration Trump. L'aventure du "Repeal and Replace » s'est, en effet, brutalement achevée au Sénat avec le vote négatif du Républicain John Mc Cain le 28 juillet 2017. Peut-on imaginer qu'un tel vote fut possible si la réforme Obama avait - par miracle - intégré l' «option publique » ? Cette défaite politique de Donald Trump, chantre de la polarisation politique, montre que la tradition politique du pragmatisme et de la conciliation n'a pas perdu toute vigueur aux États-Unis (Genieys, 2020). Elle n'est, en tout cas, pas le fait d'un «deep state »- cette bureaucratie étatique qui empêcherait l'expression démocratique - qu'il villipende. Les gardiens des politiques du secteur de la santé ne sont pas des bureaucrates de carrière mais des individus recrutés sur contrat et nommés publiquement dans ces fonctions pour accompagner le chef de l'exécutif et les parlementaires dans leur travail de gouvernement des politiques publiques. À ce titre, ces élites de gouvernement sont, en temps de polarisation politique (Pierson, Skocpol, 2007), aussi devenues les gardiennes d'une tradition réformatrice basée sur la recherche de consensus autour de certaines politiques. Dès lors, leur rôle pivot peut être difficilement ignoré dans la compréhension de la transformation du policy state américain.

William Genieys

Sciences po, CEE

william.genieys@sciencespo.fr

Mohammad-Saïd Darviche, Université de Montpellier, CEPEL said.darviche@umontpellier.fr

Brent Epperson

Université de Montpellier, CEPEL epperson@ualberta.ca

\footnotetext{
${ }^{28}$ Entretien OPERA, Washington D.C., 30 avril $2010: 7$ [http://cepel.edu.umontpellier.fr/files/2015/04/ANR-OPERAEntretien_sante_23.pdf].

${ }^{29}$ Entretien OPERA, Washington D.C., 24 mai 2010 : 6 [http://cepel.edu.umontpellier.fr/files/2015/04/ANR-OPERAEntretien_sante_10.pdf].
} 


\section{BIBLIOGRAPHIE}

Allison, G. (2006), «Emergence of Schools of Public Policy: Reflections by a Founding Dean », in Moran, M., Roin, M., Goodin, R. G. (eds), The Oxford Handbook of Public Policy, Oxford, Oxford University Press, p. 58-79.

Balogh, B. (2009), A Government out of Sight. The Mystery of National Authority in NineteenthCentury America, Cambridge, Cambridge University Press.

Beaussier, A.-L. (2012), «The Patient Protection and Affordable Care Act: The Victory of Unorthodox Lawmaking », Journal of Health Politics, Policy and Law, 37 (5), p. 743-778.

BeAussier, A.-L. (2016), La Santé aux États-Unis. Une histoire politique, Paris, Presses de Sciences Po.

BÉland, D. (2019), How Ideas and Institutions Shape the Politics of Public Policy, Cambridge, Cambridge University Press.

Benoit, C., Bezes P. (2019), «Review. The Policy State. An American Predicament. Karen Orren \& Stephen Skowronek », Public Administration, 97 (4), p. 962-964.

BriLl, S. (2015), America's Bitter Pill. Money, Politics, Backroom Deals, and the Fight to Fix our Broken Healthcare System, New York (N.Y.), Random House.

Brown, L. D. (1983a), Politics and Health Care Organization. HMO's as Federal Policy, Washington D.C, The Brookings Institution.

Brown, L. D. (1983b), New Policies, New Politics: Government's Response to Government Growth, Washington D.C., Brookings Institution.

Brown, L. D. (1985), «Technocratic Corporatism and Administrative Reform in Medicare », Journal of Health Politics, Policy and Law, 10 (3), p. 579-599.

Brown, L. D. (2019), «Single-Payer Health Care in the United States: Feasible Solution or Grand Illusion », American Journal of Public Health, 109 (11), p. 1506-1510.

DARVICHE, M.-S., GENIEYS, W. (2018), «Quand le Léviathan se fait Phoenix : les voies d'une résurrection annoncée de l'État ? », Gouvernement et Action publique, 7 (1), p. 113-122.

DARViChe, M.-S., GenieYs, W., HoEFFler, H., JoANA, J. (2013), «Des “long timers" au sommet de l'État américain. Les secteurs de la Défense et de la Santé (1988-2010) », Gouvernement et action publique, 2 (1), p. 10-38.

Derthick, M. (1979), Policymaking for Social Security, Washington D.C., The Brookings Institution Press.

EPPERSON, B. (2019), «Challenging Narratives to Neoliberalism in Media Representations of American Health Reform: the Massachusetts and Utah Cases (2002-2011) », in KellogG, C., HARDER, L. (eds), Neoliberal Contentions, Toronto, University of Toronto Press.

Genieys, W. (2010), The New Custodians of the State. The Programmatic Elites in French Society, Rutgers N.J., Transactions Books.

GENIEYS, W. (2020), Gouverner à l'abri des regards. La réussite de l'Obamacare, Paris, Presses de Sciences Po.

Genieys, W., HASSENTEUfEl, P. (2012). «Qui gouverne les politiques publiques ? Par-delà la sociologie des élites », Gouvernement et action publique, 1 (2), p. 89-115.

Genieys, W., Hassenteufel, P. (2015), «The Shaping of the New State Elites. Healthcare Policymaking in France Since 1981. » Comparative Politics, 47 (3), p. 280-295.

GENIEYs, W., JoAnA, J. (2015), «Bringing the State Elites Back in ? Les Gardiens des politiques de l'État en Europe et aux États-Unis », Gouvernement et action publique, 4 (3), p. 57-80. 
Hacker, J. S. (2010), «The Road to Somewhere: Why Health Reform Happened », Perspectives on Politics, 8 (3), p. 861-876.

Hassenteufel, P., Le Galès, P. (2018), «The Academic World of French Studies: Training, Teaching and Researching », in HALPERn, C., Zitoun, Ph. (eds), Policy Analysis in France, Bristol UK, Polity Press, p. 295-312.

Hassenteufel, P., Genieys, W., Smyrl, M., Moreno, J. (2010), « Programmatic Actors and The Transformation of European Health Care States », Journal of Health Politics, Policy and Law, 35 (4), p. 517-538.

HeClO, H. (1977), A Government of Strangers. Executive Politics in Washington, Washington D.C., The Brookings Institution.

HeClo, H. (1978), « Issues Networks and the Executive Establishment », in BEER, S. H. et al. (eds), The New American political System, Washington D.C., American Institute Entreprise, p. 87-124.

Heclo, H. (1984), «In a Search of Role. Amerca's Higher Civil Services », in Suleiman, E. N. (ed.), Bureaucrats and Policy Making. A Comparative Overview, New York \& London, Holmes \& Meier, p. 8-34.

Jacobs, N. F., King, D., MiLKIS, S. M. (2019), «Building a Conservative State: Partisan Polarization and the Redeployment of Administrative Power », Perspectives on Politics, 17 (2), p. 453-469.

Joyce, P. G. (2011), The Congressional Budget Office. Honest Numbers, Power and Policymaking, Washington D.C., The Georgetown University Press.

LEPONT, U. (2014), Façonner les politiques aux marges de l'État. Le rôle des experts dans les réformes de la protection maladie aux États-Unis (1970-2010), Thèse de doctorat en science politique, Université de Montpellier.

LEPONT, U. (2016a), «Les experts les plus consultés aux États-Unis : 1'hypothèse d'une "périadministration" : le cas des politiques de protection maladie », Revue française de science politique, 66 (6), p. 887-913.

LEPONT, U. (2016b), « De Clinton à Obama : effets d'apprentissage et filtrage des alternatives : la convergence des experts sur la réforme de santé aux États-Unis (1993-2010) », Gouvernement et action publique, 5 (3), p. 9-31.

McDonough, J. E. (2012), Inside National Health Reform, Berkeley (Calif.), California University Press.

Marmor, T., Oberlander, J. (2011), « The Patchworh : Health Reform, American Style », Social Science and Medicine, 72 (2), p. 125-128.

Mettler, S. (2011), The Submerged State. How Invisible Government Policies Undermine American Democracy, Chicago (Ill.), Chicago University Press.

Mizruchi, M. S. (2013), The Fracturing of the American Corporate Elite, Cambridge, Harvard University Press.

Oberlander, J. (2003), The Political Life of Medicare, Chicago (Ill.), Chicago University Press.

OBERlander, J. (2019), «Lessons From the Long and Winding Road to Medicare for All», American Journal of Public Health, 109 (11), p. 1497-1500.

OrRen, K., Skowroneck, S. (2017), The Policy State, Cambridge, Harvard University Press.

Pierson, P. (1994), Dismantling the Welfare State? Reagan, Thatcher, and the Politics of Retrenchment, Cambridge, Cambridge University Press.

Pierson, P., Skocpol, T. (eds) (2007), The Transformation of American Politics: Activist Government and the Rise of Conservatism, Princeton (N.J.), Princeton University Press.

PutNAM, R.D. (1977), «Elite transformation in Industrial Advanced Societies. An Empirical Assessment of the Theory of Technocracy » Comparative Political Studies, 10 (3), p. 383-411. 
RodwIN, V. G. (1985), «Les Health Maintenance Organizations (HMO) aux États-Unis : un bilan », Politiques et management public, 3 (4), p. 39-85.

SElzNICK, Ph. (1957), Leadership in Administration. A Sociological Interpretation, Berkeley (Calif.), California UniversityPress.

Steinmo, S. (2010), The Evolution of Modern States. Sweden, Japan, and the United States, Cambridge, Cambridge University Press.

Suleiman, E. N. (2003), Dismantling the Democratic States, Princeton (N.J.), Princeton University Press. 